الدركاة وعلاقتها بموقع كتلة المدخل بالواجهة من منظور علوم الطاقة

The Durka and its relation to the entrance location in the elevation from the point of view of energy science

أ.دأد أشرف حسين ابراهيم

أستاذ التصميم البيئى بقسم التصميم الداخلى والأثاث ـ كلية الفنون التطبيقية جامعة حلوان

Prof. Ashraf Hussin

Professor of Interior Design and Furniture, Faculty of Applied Arts, Helwan University ashrahus@gmail.com

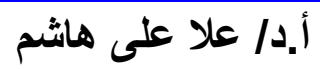

أستاذ تصميم المنثأت السياحية ووكيل الكليه لثئون اللاراسات العليا والبحوث ـ كلية الفنون التطبيقية جامعة حلوان

Prof. Ola Hashem

Professor, Department of Interior Design and Furniture, Faculty of Applied Arts, Helwan University

0.a.hashem@hotmail.com

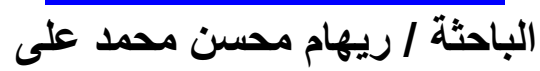

باحثة دكتوراة بقسم التصميم الداخلي والأثاث ـ كلية الفنون التطبيقية جامعة حلوان

Researcher.Riham Mohsen Mohamed Ali

PHD. Researcher at interior design and furniture department, Helwan University riham_mohsen@hotmail.com

$$
\text { الأستاذ المساعد بقسم العمارة , جامعة مصر الصاوير الدولية }
$$

Assist. Prof. Mohamed Samir El-Sawy

Doctor of architecture department, MIU University

mssawy@yahoo.com

\begin{abstract}
ملخص البحث :-

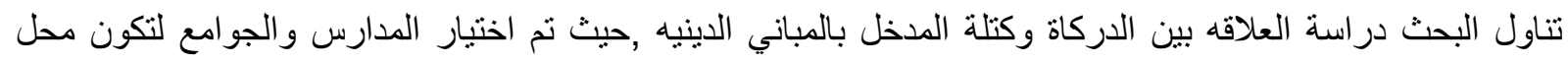
الدراسه التحليلية وذلك لكونها أكثر الأماكن روحانيه وطاقة منظمة بناء على توجيهها إلى الكعبة المشرفة , حيث تم تم التحليل

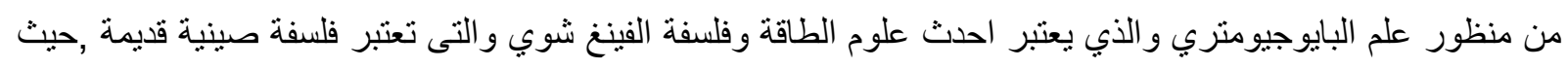

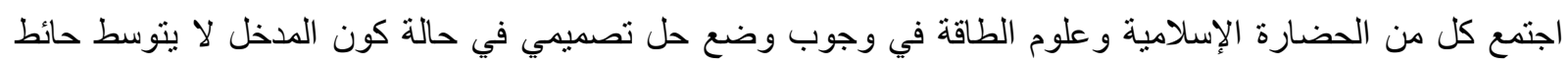

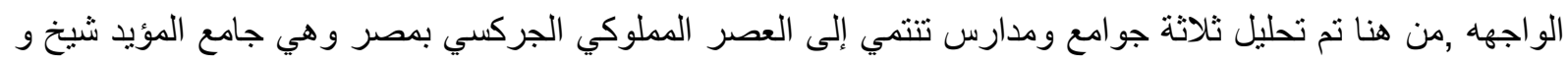

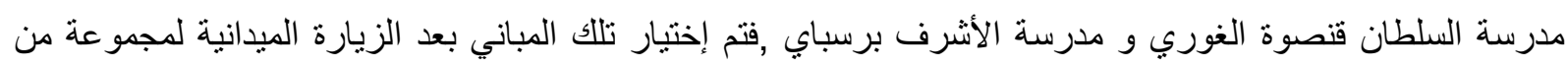

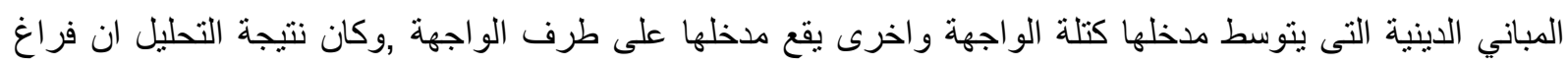

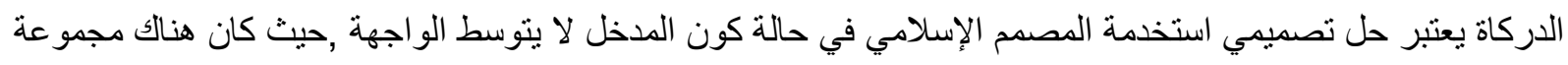

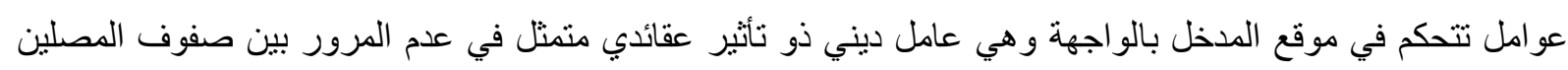

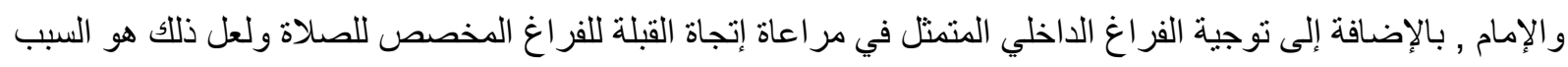

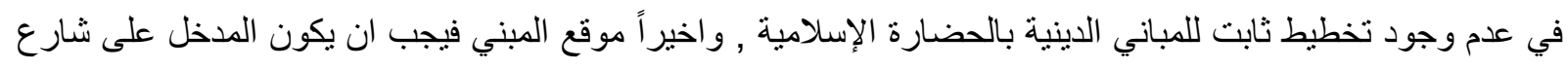

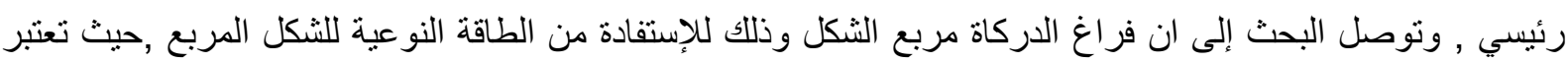


الدركاة فر اغ يربط بين المدخل وباقي اجز اء المبني الديني من خلال بابين يتوسط كل منهما الحائط الجنوبي و الثمالى لحائط

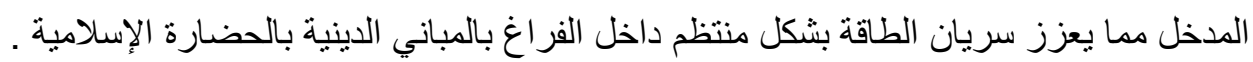

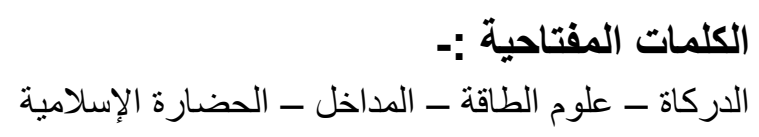

\begin{abstract}
:-
The study examined the relationship between the Durka and the entrance block of religious buildings. Schools and synagogues were chosen as the subject of analytical study because they are the most spiritual places and the most organized energy based on their orientation to the honorable Kaaba. The analysis was done from the perspective of biogeometry, which is the latest in energy science and the philosophy of feng shui, which is an ancient Chinese philosophy. Both Islamic civilization and energy sciences have come together in order to develop a design solution in the event that the entrance does not mediate the wall of the facade. Three jewels and schools belonging to the Mamluki-Jarksi era in Egypt, namely the Al-Maid Sheikh, the Sultan Qunsoua Al-Ghuri School and the Ashraf Pressbay School, were analysed.These buildings were selected after the field visit of a group of religious buildings, the entrance to which is located on the front block and the entrance to which is located on the tip of the facade. The result of the analysis was that the darkening vacuum was a design solution used by the Islamic designer.
\end{abstract}

\title{
Key Words:-
}

The Durka - Energy Science - Entrances - Islamic Civilization

الحضارة الإسلامية مصدر للإلهام و الإبدع ,ذللك لما تحمله من معاني رمزية وضمنية ,فلم بعتمد المصمم الإسلامي على السطحيه والثكل الخارجي ,بل كان لكل تصميم وتتكيل دلاله رمزيه , و أبعاد فلسفية ولعل ذلك ما سيتم تسليط الضوء ولهيه عليه

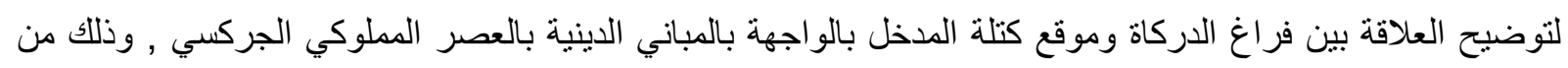

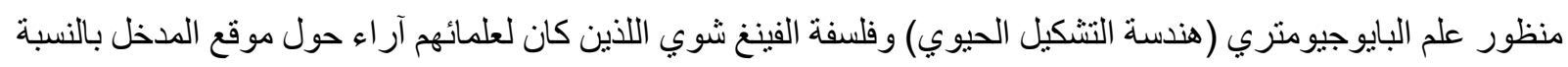
للحائط وحلول مقترحة في حالة الوضع الغير مثالي وذلك لتعزيز الطاقة الإيجابية داخل الفراغات المختلفة ,سيتم ذلك من

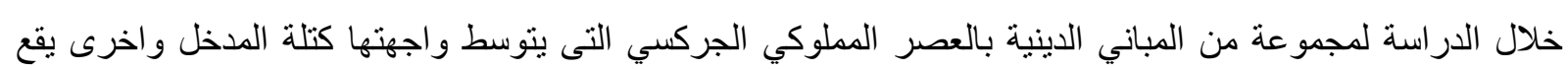

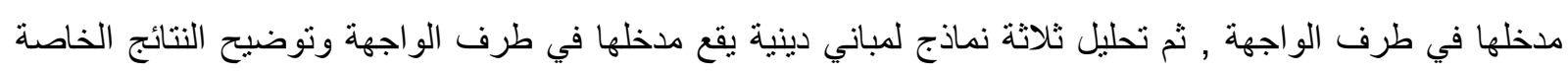
بالتحليل . مشكلة البحث :على ضوء ذللك يمكن صياغة مشكلة البحث البحث في الأسئلة التالية : - - ما هي علاقة فراغ الدركاة بموقع المدخل بالواجهة بالمباني الدينية ؟

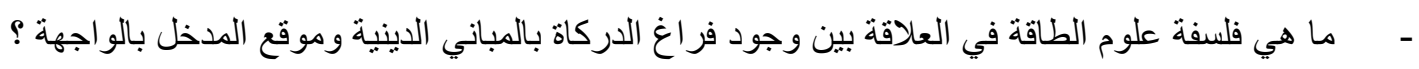
هدف البحث :يهدف البحث إلى الكثف عن فلسفة علوم الطاقة في العلاقة بين فراغ الدركاة وموقع المدخل بو اجهة المباني الدينية 
- يفترض البحث ان وجود دركاة بالمباني الإسلامية مرتبط بموقع المدخل بالو اجهة .

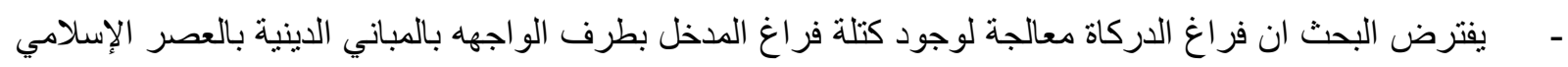
المملوكي الجركسي . اهمية البحث :ترجع اهمية البحث إلى تسليط الضوء على البعد الضمني لا السطحي لوجود فراغ الدركاة بالمباني الإسلامية حدود البحث :الحد الزماني : عصر المماليك بالحضارة الإسلامية

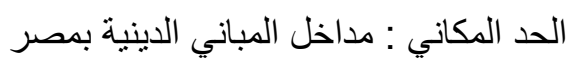

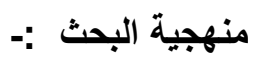
يتبع البحث المنهج الوصفي التحليلي

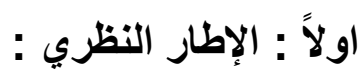

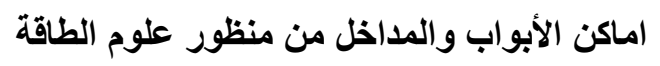

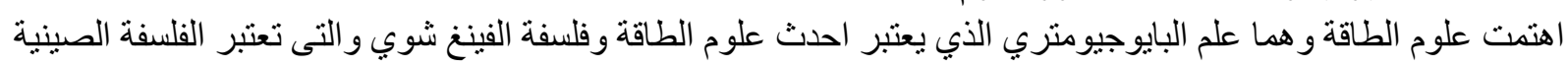
القديمة, بدر اسة بعض العناصر و المفردات المعمارية ورصد افضل نتائج تساعد على تنظيم الطاقة داخل الفر اغات المختلفة , حيث يتعامل علم البايو جيو متري مع طاقة النظام الكوني ويعمل على إعادة الإتزان إليها من خلال در اسة الأشكال الهندسية

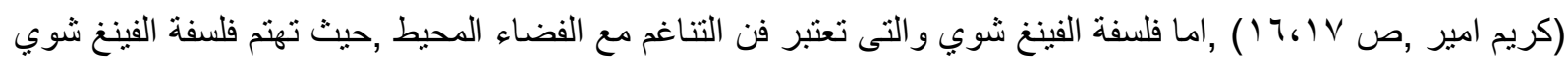
بملاحظة علاقه الأشياء ببعضها البعض و عناصر الطبيعة والكونيه وتأثثرها على الإنسان (Michael Erlewine) ,

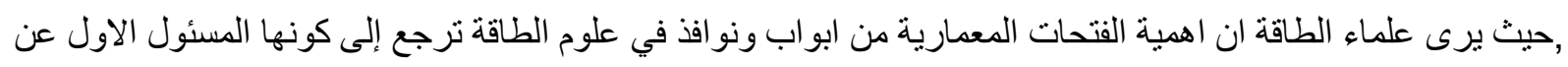

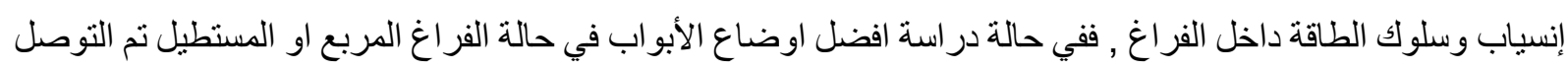
الى ان افضل وضع هو كون الباب في وسط الحائط (تحليل الباحثة , من منطلق دورة تدريبية في علم "البايوجيومثري")

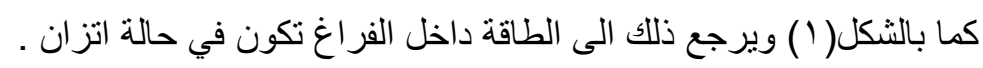

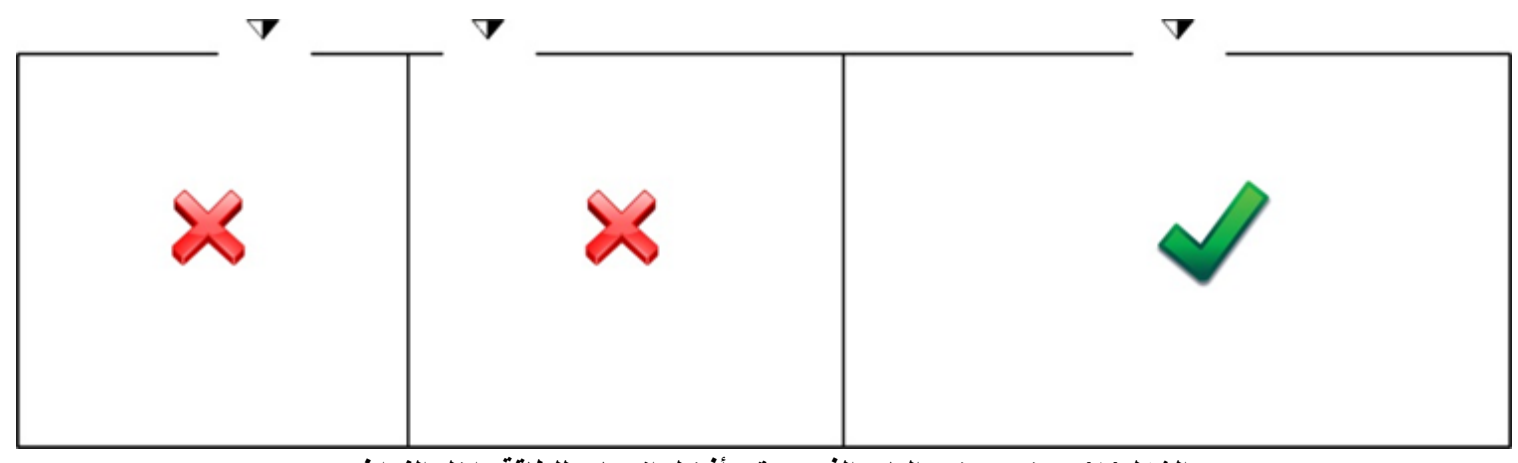

الثكل( ا ) يوضح وضع الباب الذي يحقق أفضل انسياب للطاقة داخل الفراغ .

المصدر:اعداد الباحثة. النئ.

ولكن في حالة كون المدخل جانبي يقدم علم الطاقة بعض الحلول عن طريق التصميم الداخلى لإعادى الإتزان داخل الفراغ

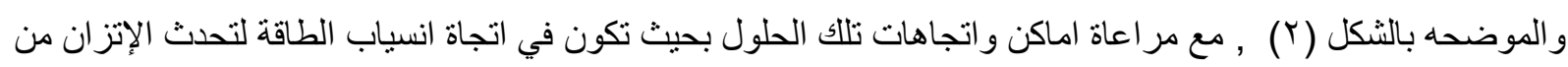
خلال تغير تدفقها و انتظامها. 
اكتوبر ا T.

مجلة التراث والتصميم ـ المجلد الاول ـ العدد الخامس

فى حالة كون الباب على طرف احد الحوائط يتم عمل تجليدة امام الباب لتأثر على طبيعة سريان الطاقة وتعيد لها الإتزان

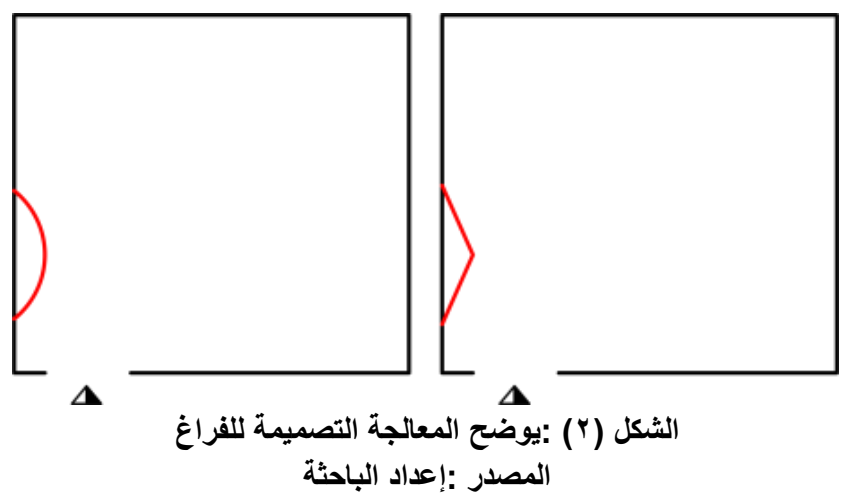

ويمكن تحقيق نفس النأثثر السابق ذكره من خلال وضع قطعة أثاث تتخذ الثكل المطلوب مع مر اعاة الخامة واللون

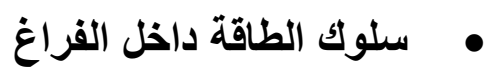
لإدراك السبب في تحديد منتصف الحائط هو افضل مكان لوضع المدخل يجب ان نذكر كيف يتم انتشار ومسار الطاقة في الفراغ ,حيث انه يثابه مسار وانتشار الهو اء حيث ينساب في خط مستقيم في اتجاه التبار محدثا مجالين احدهم اتجاة اليمين و الآخر إتجاة يسار خط اتجاة انسياب الطاقة كما بالثكل (ץ), بحيث يكون المجال يمين خط الاتجاه في اتجاه عقارب الساعة

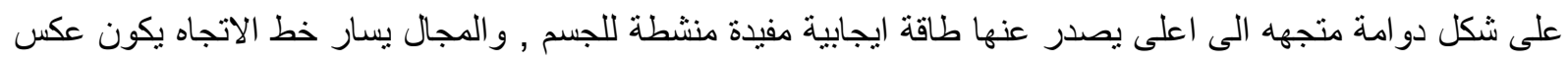
lbrahim Karim,pg ) اتجاه عقارب الساعة ومتجة الى اسفل يصدر عنة طاقة سالبة تؤدي الى خمول الجسه . $(69,70,71$

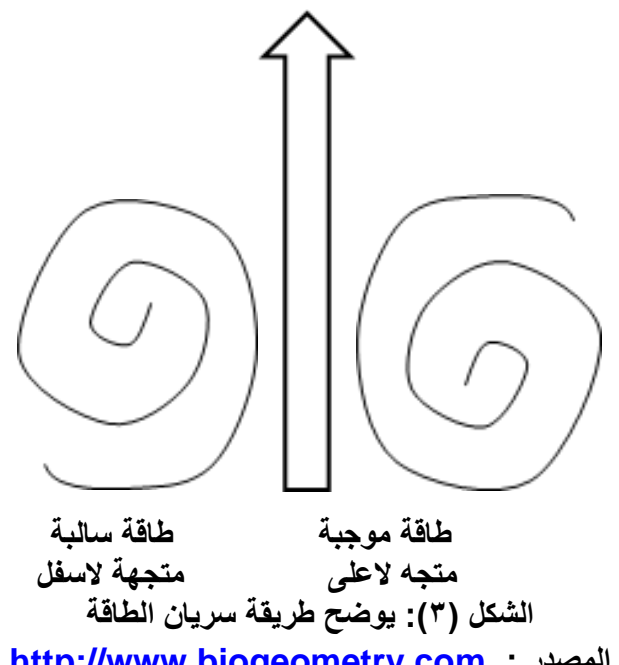

http://www.biogeometry.com : المصدر : برضكل 


\section{موقع المدخل في المباني الاينية وتأثيرة على التصميم الأخلي}

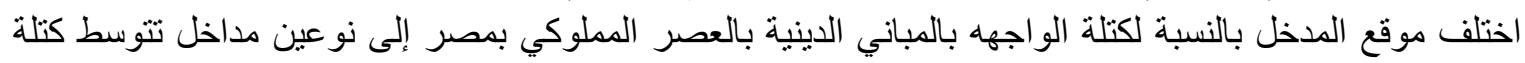

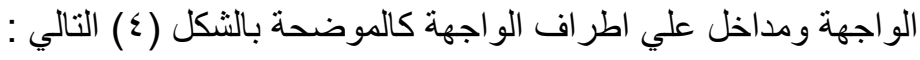

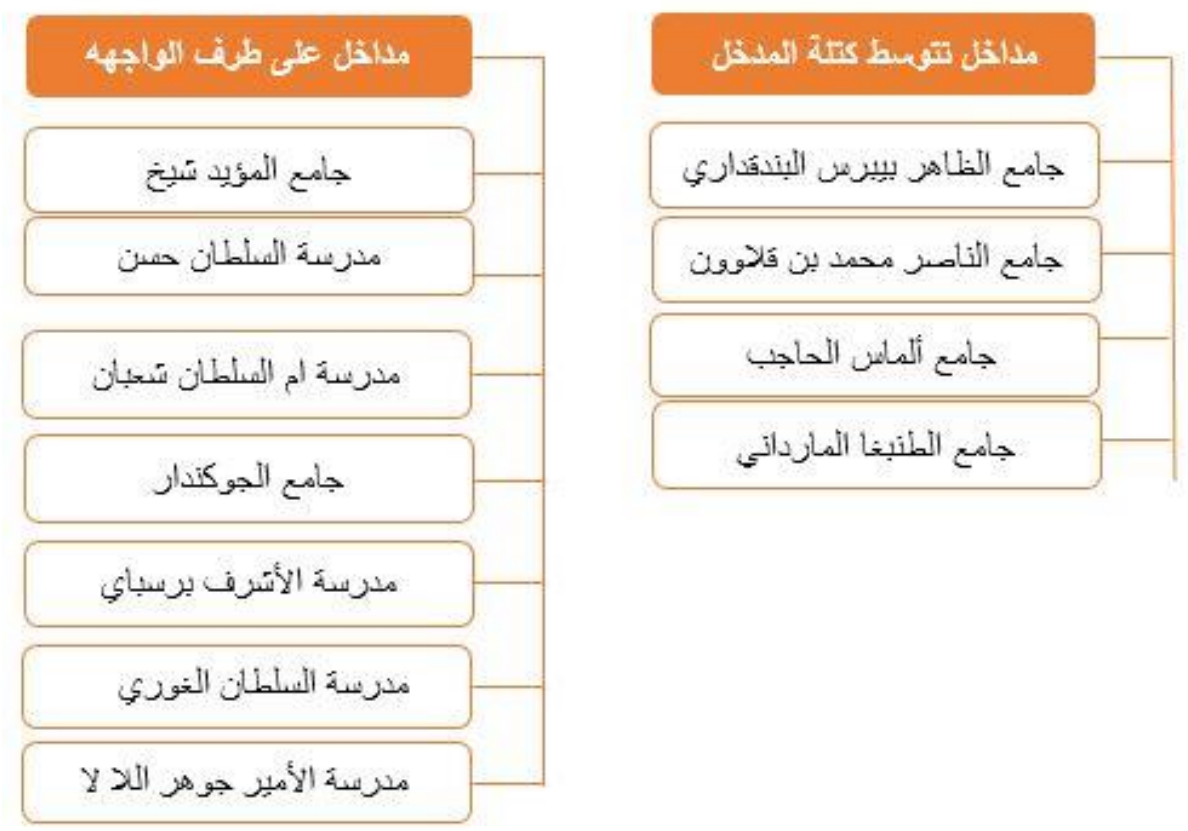

الثكل (؛ ): يوضح الانواع المختلفة للمداخل حسب موقعها بكتلة المدخل

\section{• العوامل المؤثرة في موقع المدخل بالمباني الاينية}

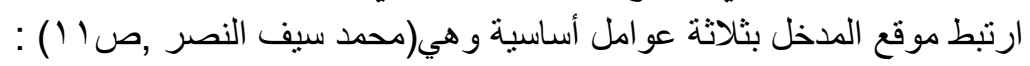

- اولاً : العامل الديني : حيث خضع اختيار مكان الدذخل إلى التأثير العقائدي والمتمثل في عدم المرور بين صفوف المصلين أو أمامهم .

- ثانياُ : توجية الفراغ الداخلي : حيث كان مر اعاة إتجاة القبلة للفر اغ الداخلى المخصص للصلاة سبب في عدم وجود

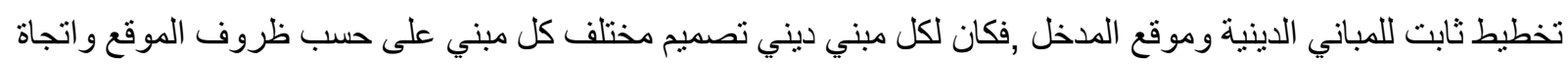

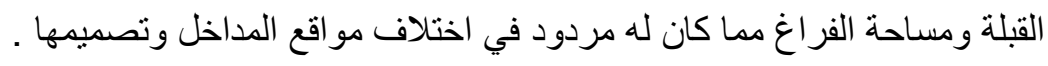
- ثالثأ : موقع المبني الايني : حيث ارتبط موقع المدخل بالواجهة وبالطريق الرئيسي لذا تعددت تصميمات المباني الدينية مع الحفاظ على العناصر الرئيسية للو اجهة وهي القبة و المأذنة وكتلة المدخل .

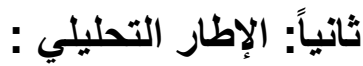
• سوف يتم تحليل مداخل مجموعة من المباني الدينية بالعصر الملولوكي الجركسي بالحضارة الإسلامية وإستنتاج علاقة المخل الجانبي بفراغ بالدركاة .

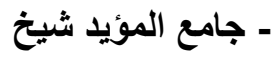
الجامع الذي انثأه السلطان المؤيد ابو النصر شيخ الدحمودي الجركسي ,والو اقع بشارع المعز لدين الدين الله ملاصقاً لباب زويلة

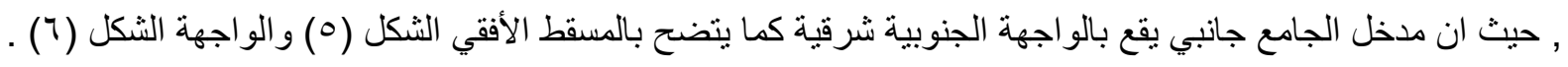




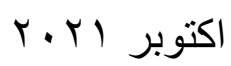

مجلة التراث والتصميم - المجلد الاول ـ العدد الخامس

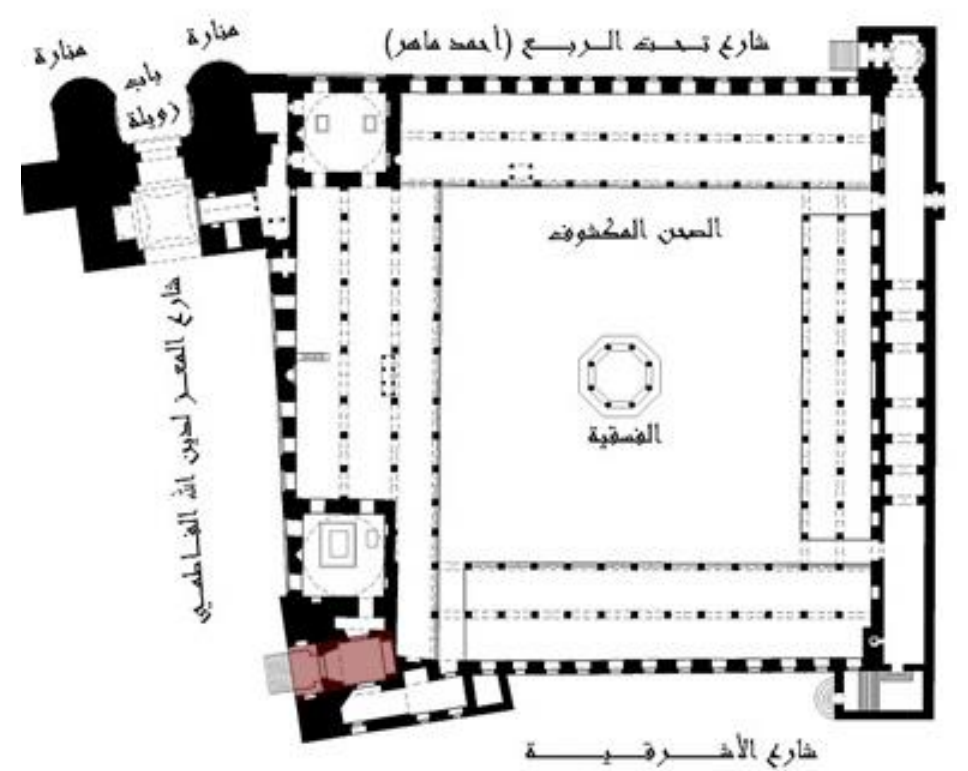

الثكل (0) :يوضح المسقط الأفقي لجامع المؤيد شيخ

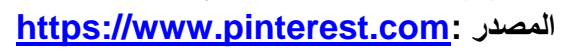

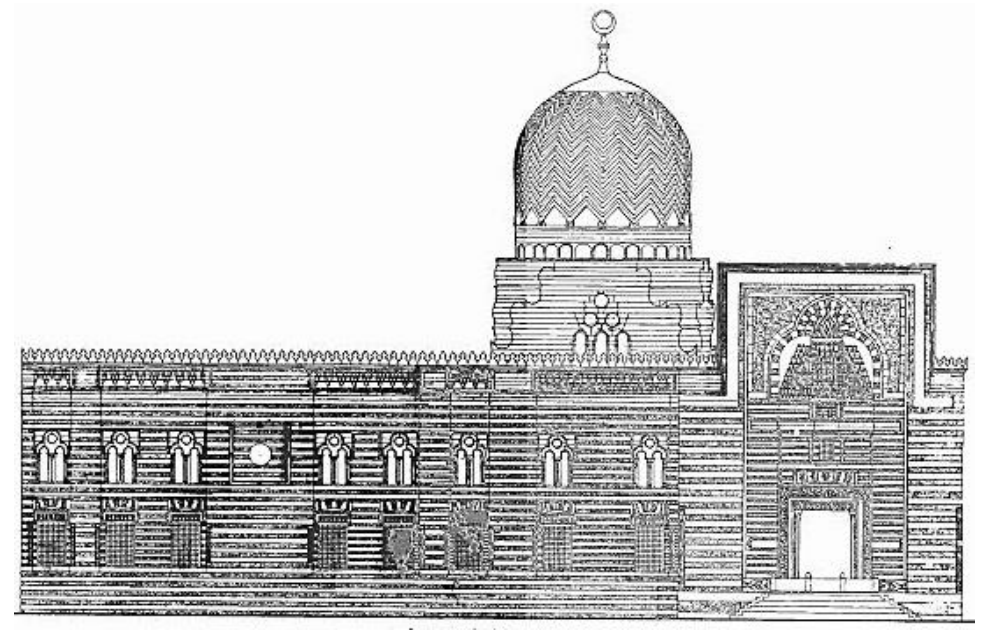

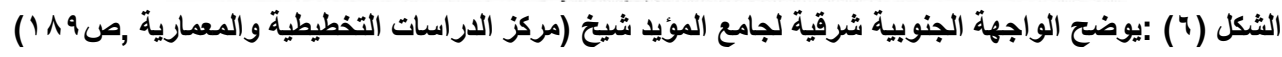

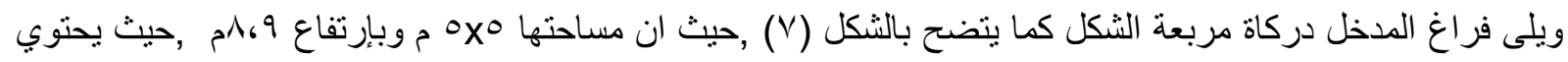

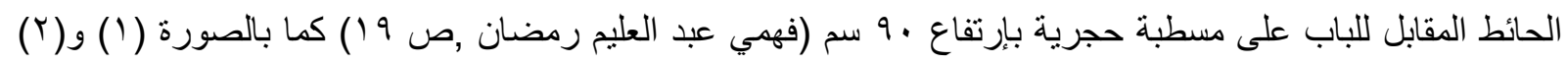
يغطي الدركاة قبو متقاطع Cross Vault كما بالصورة (r) مغطي بالمقرنصات كما بالصورة (ع) ) .

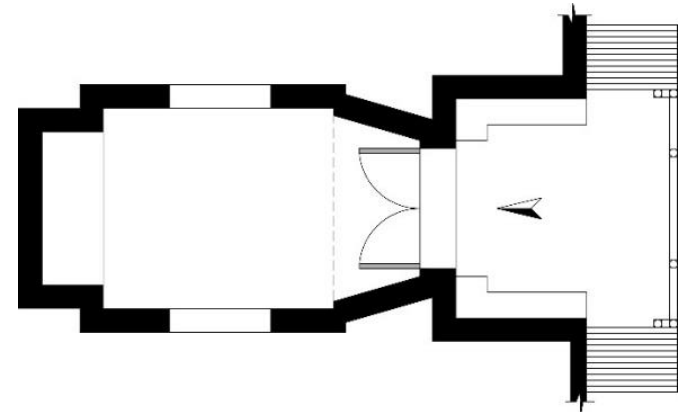

الثكل (V) :يوضح المسقط الأفقي لمدخل ودركاة جامع المؤيد شيخ المصدر : تصميم الباحثة 


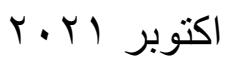

مجلة التزاث والتصميم ـ المجلد الاول ـ العدد الخامس
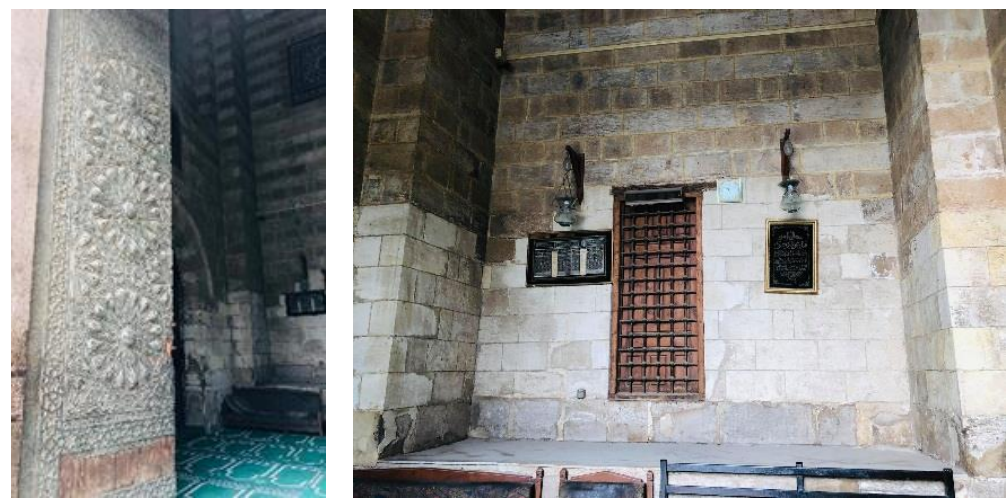

الصورة (1) و (ץ): الاركاه من الاخل والمسطبة بالحائط المقابل للمدخل

المصدر: تصوير الباحثة

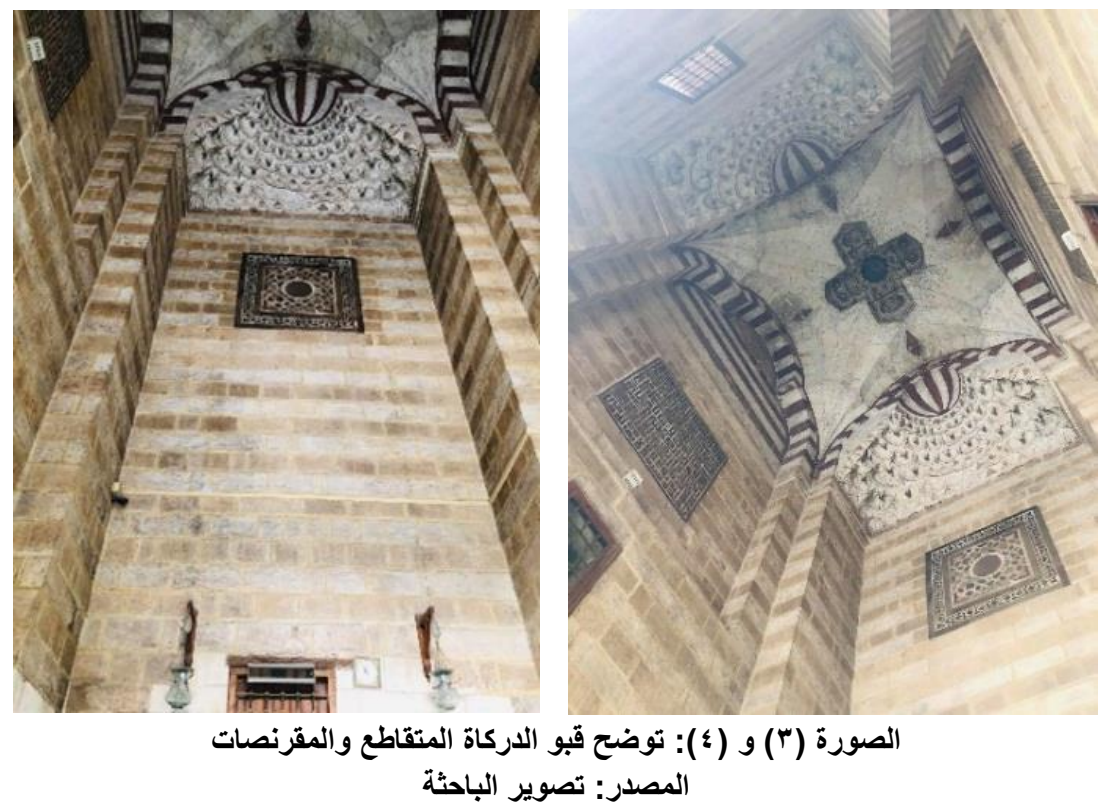

يحتوي الحائط الأيمن و الأيسر بالدركاة على بابين، الأيمن يؤدي إلى دهليز ومنه إلى صحن الجامع، اما الباب الأيسر يؤدي

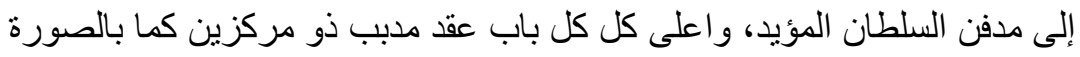

(7) $(0)$
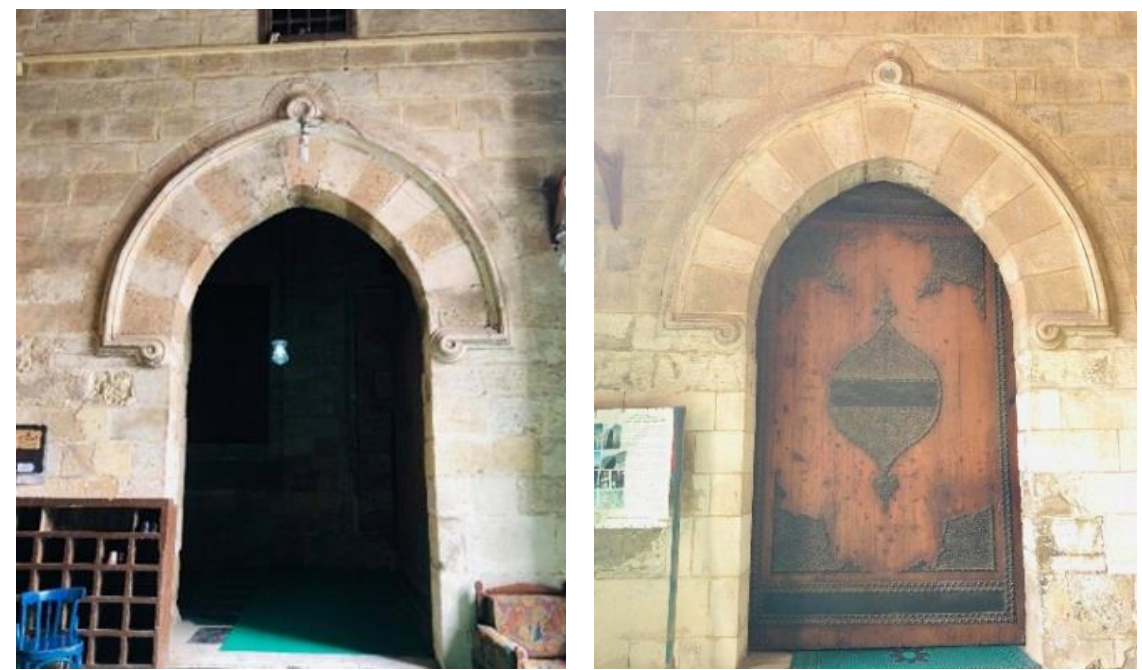

الصورة (0) و(آ) : توضح العقود المدبية ذات المركزين بباب الدهليز والمدفن

المصدر : توصير الباحثة البركئ 
البابين من الخشب ذو دلفة واحدة يتوسط الدلفة بخارية بيضاوية من النحاس يتوسطها شريط من الكتابة بخط النسخ , كما

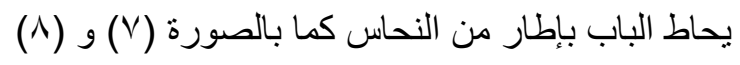
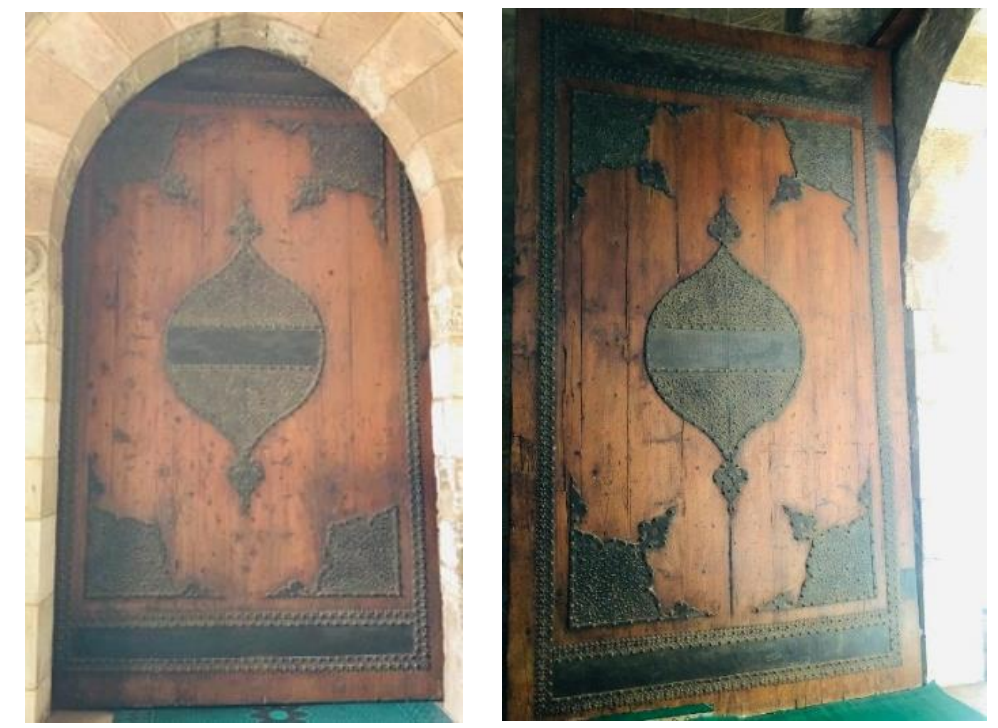

الصورة (V) و(^) : توضح العقود المدبية ذات المركزين بباب الاهليز والمدفن المصدر : توصير الباحثة العركئ

- مدرسة السلطان قنصوة الغوري

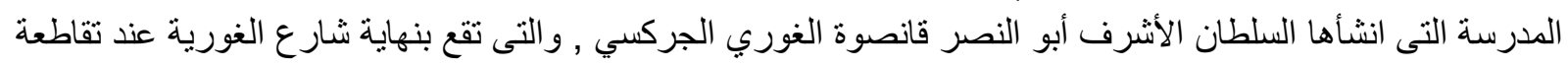

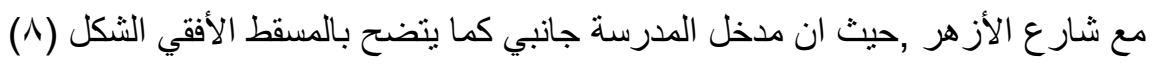

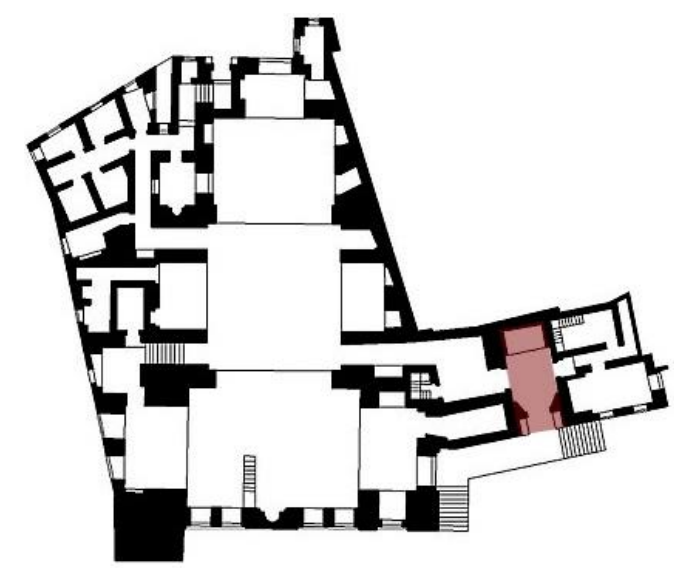

الثكل (^) :يوضح المسقط الأفقي لمدرسة قانصوة الغوري موضح بة فراغ الاركاة https://www.pinterest.com: المصدر

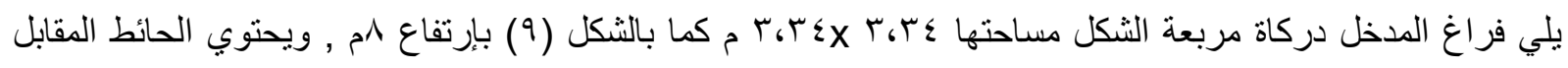

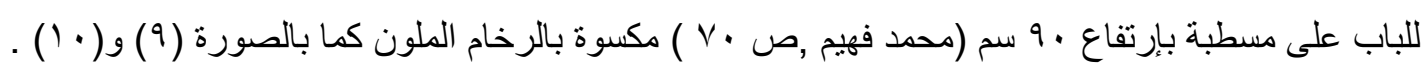


اكتوبر ا T.

مجلة التراث والتصميم ـ المجلد الاول ـ العدد الخامس

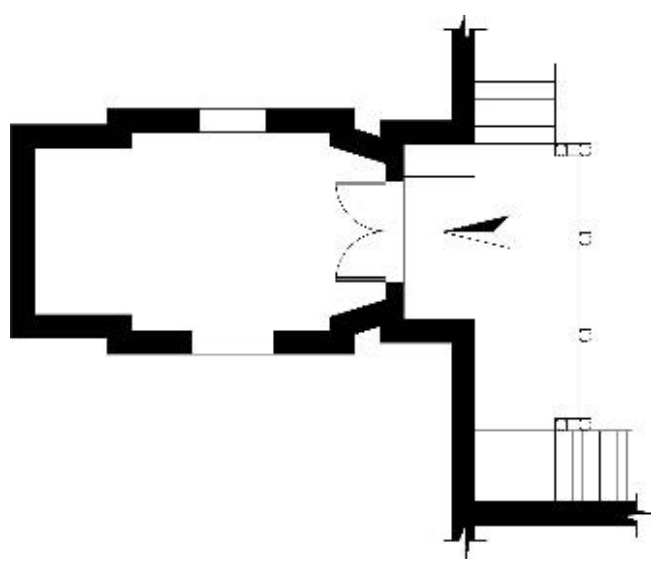

الثكل (9) : يوضح المسقط الأفقي لمدخل ودركاة مدرسة السلطان قانصوة الغوري

المصدر : تصميم الباحثة مثلة

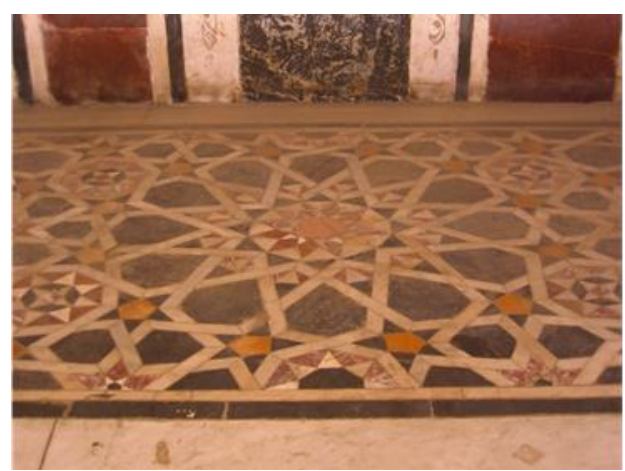

الصورة ( (1) توضح ارضية المسطبة المكسوة بالرخام الملون المصدر : تصوير الباحثة

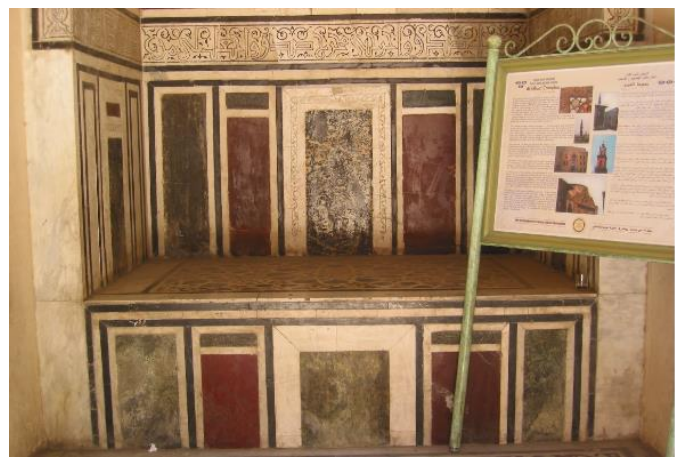

الصورة (9 ) توضح حوائط المسطبة المكسوة بالرخام الملون المصدر : تصوير الباحثة لمكئة

يتوسط الحائط الأيمن والأيسر للاركاه فتحة معقودة بعقد مدبب لباب ذو دلفة واحدة يوصل الباب الأيسر من المدخل إلى الدهليز ومنة إلى صحن المدرسة و الإيوانات والباب الأيسر يوصل إلى دهليز الخلوه كما يتضح بالصورة (1 (1) و (ب ( ).
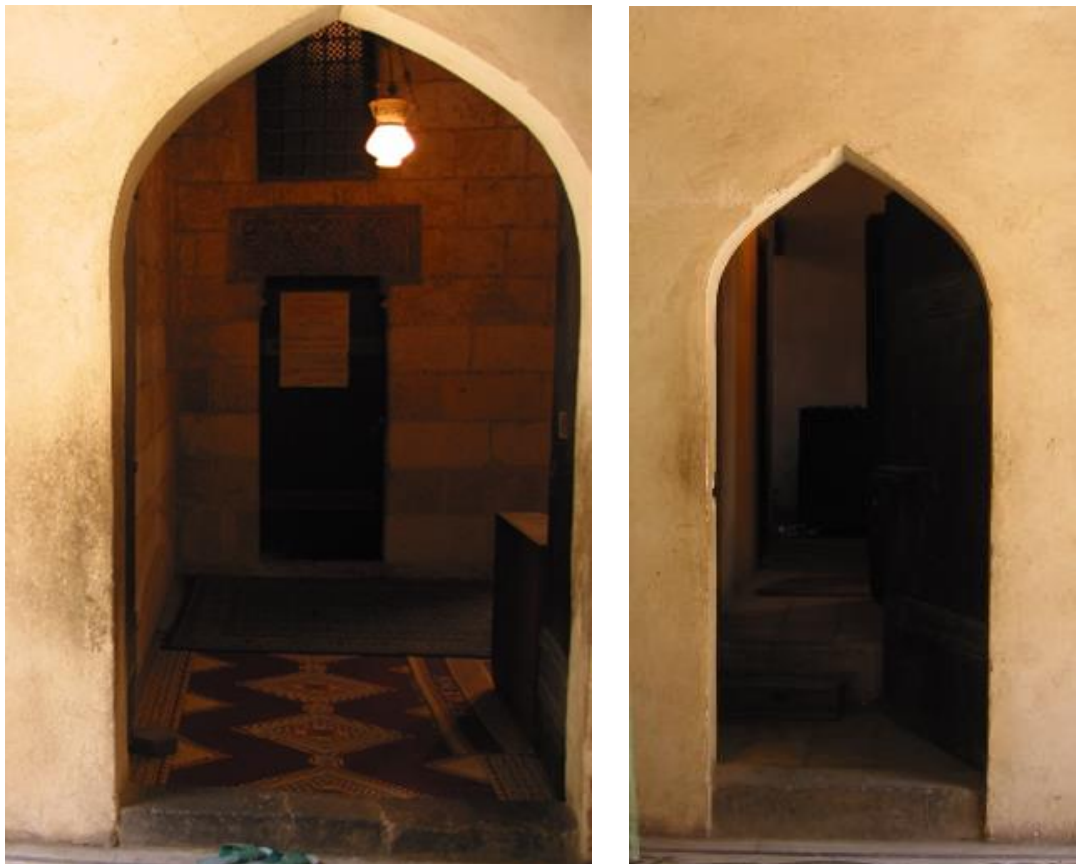

الصور (1') و (Y I I) : توضح الفتحات المعقودة بحوائط الدركاه

المصدر : تصوير الباحثة 
تسقق الدركاه بشر ائح خثبية مستوية مزخرفة عددها ب ا شريحة , ويربط السقف بالحوائط إطار خشبي مزخرف بشريط

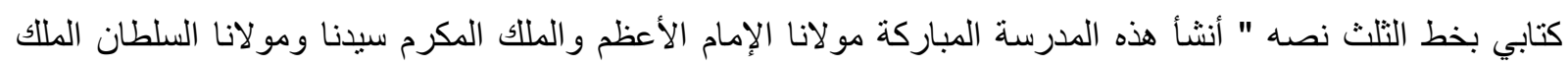

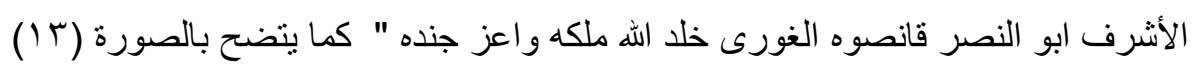

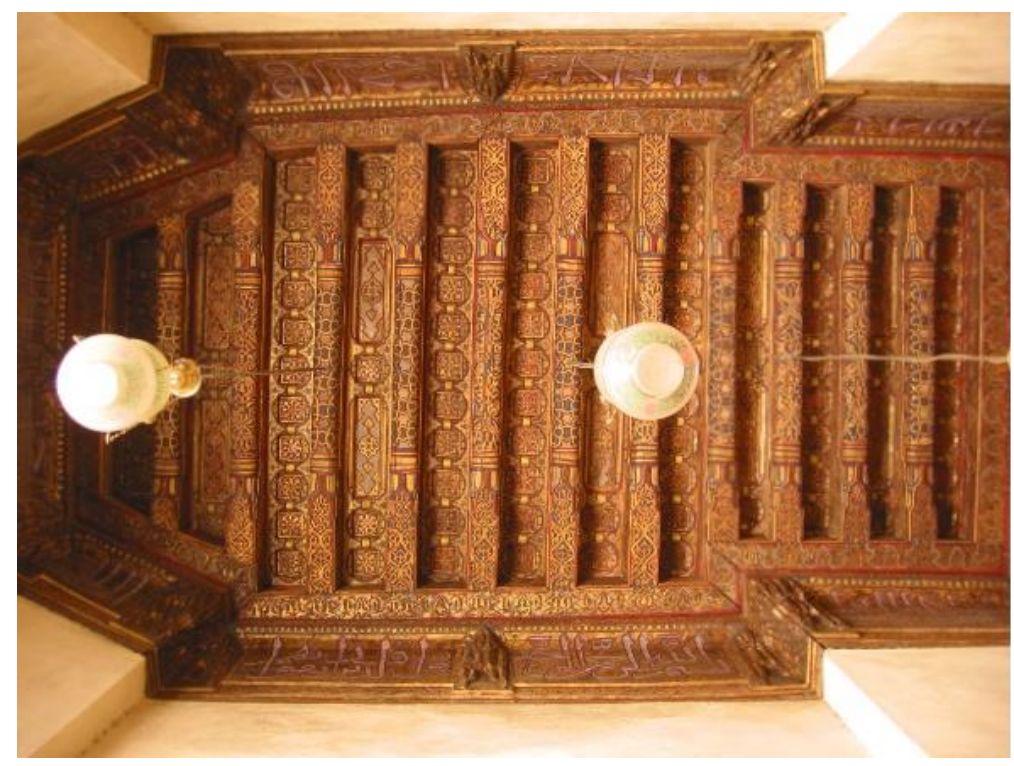

الصور (T (1) ) : توضح السقف الخشبي والثريط الكتابي بالاركاه

المصدر : تصوير الباحثة

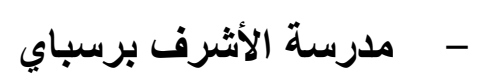

المدرسة التى انشأها السلطان الملك الأشرف سيف الدين أبو النصر برسباي ,والتى تقع بشارع المعز لدين الله عند تقاطعة

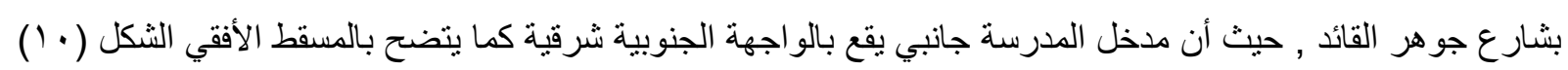

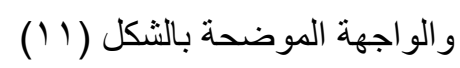

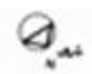

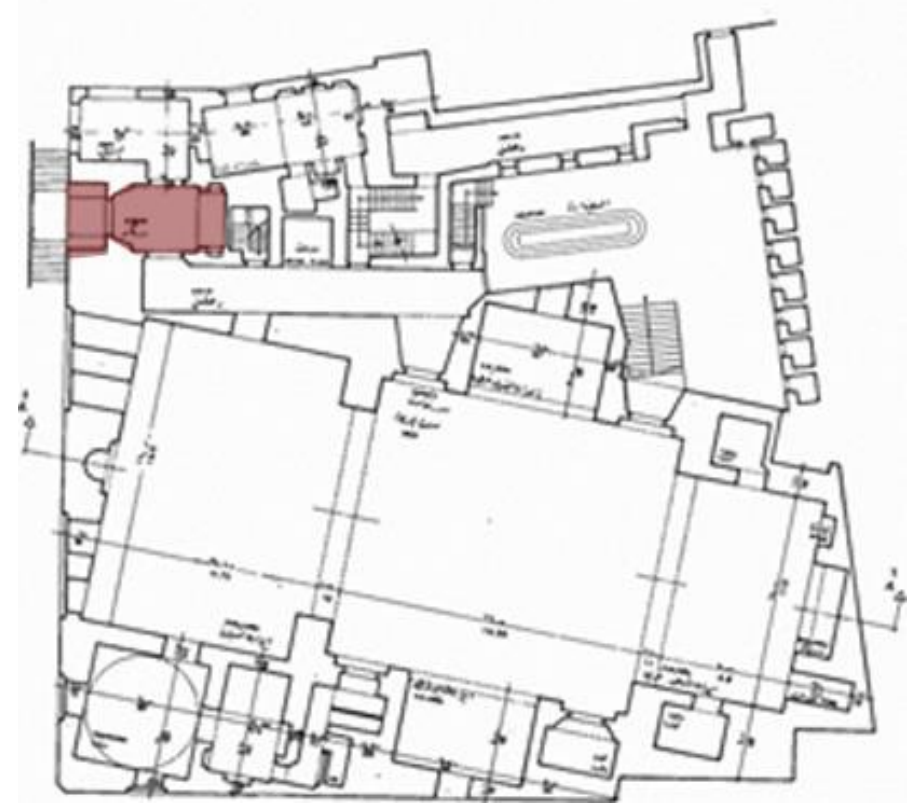

الشكل ( · () :يوضح المسقط الأفقي لمدرسة الأشرف برسباي (مركز الاراسات التخطيطية و المعمارية ,صه 9 (1) 


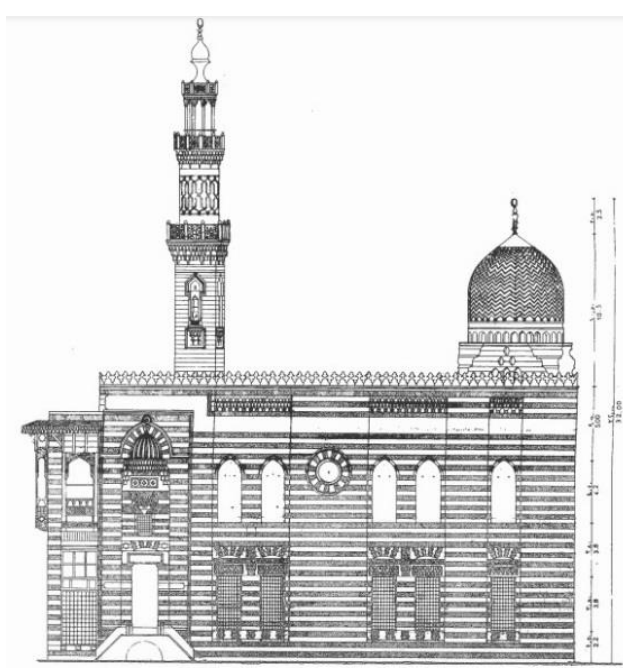

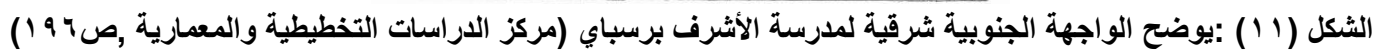

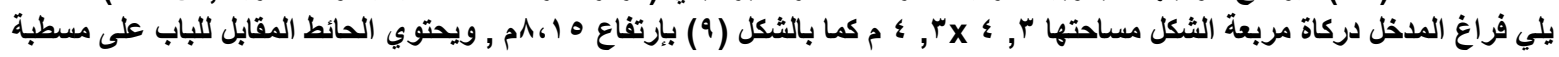

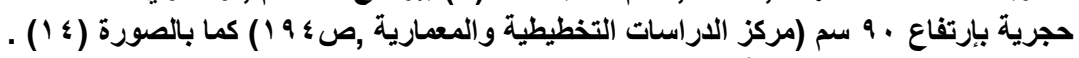

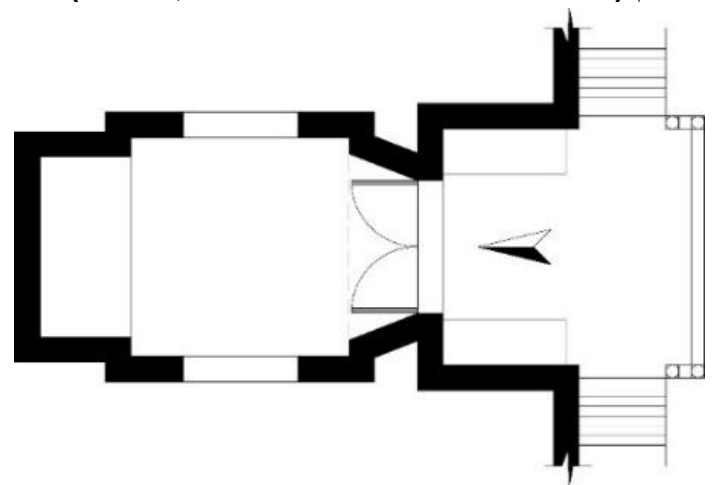

الثكل ( ) ) :يوضح المسقط الأفقي لمدخل ودركاة مدرسة الأشرف برسباي

المصدر : تصميم الباحثة المانة

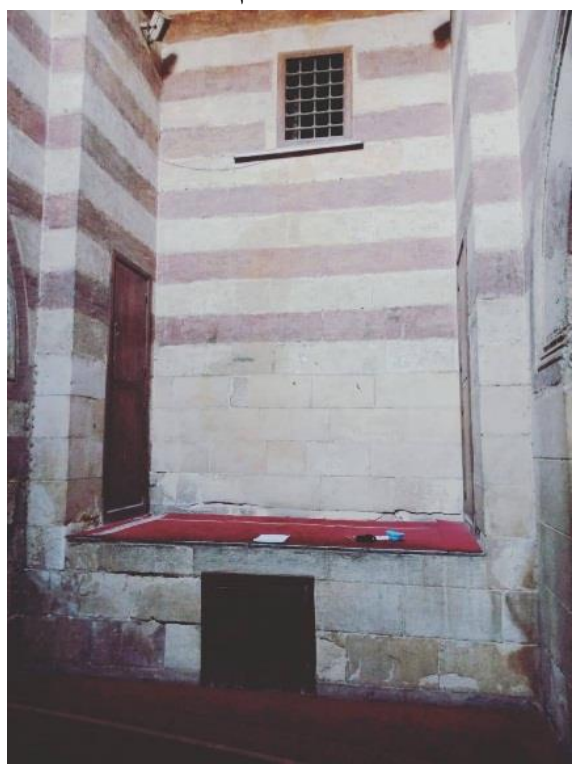

الصورة (؛ 1) : الاركاه من الداخل و المسطبة بالحائط المقابل للمدخل

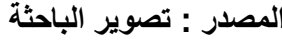




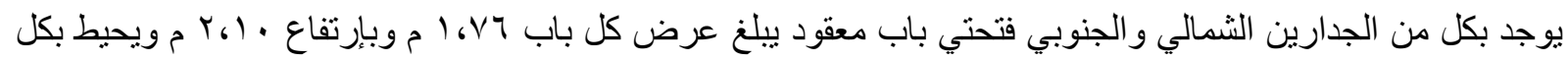
فتحة جفت بسيط ذو ميمة كما يتضح بالصورة (10) , يغطي الدركاة سقف خشبي مسطح كما بالصورة (1 (1)
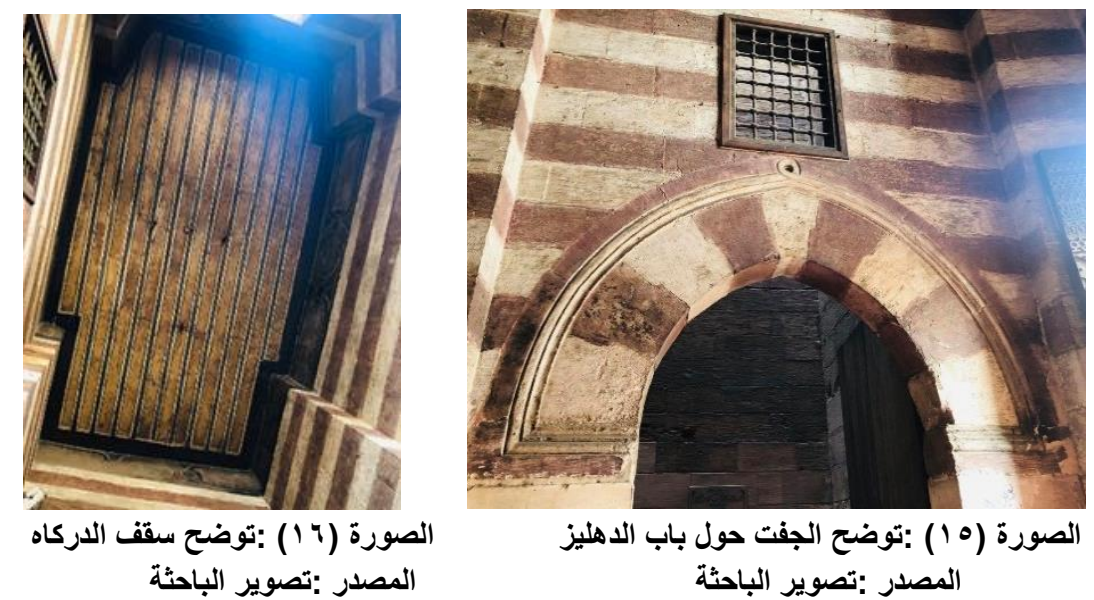

نتائج البحث

- - يرتبط وجود الدركاة بفراغ المدخل القائم بطرف الواجهة بالمباني الدينية بالعصر المملوكي الجركسي بمصر . - اتخذت الدركاة بالمباني الدينية الثكل المربع وذللك للإستفادة من الطاقة النو عية و الرمزية للشكل المربع , حيث اعتبرت

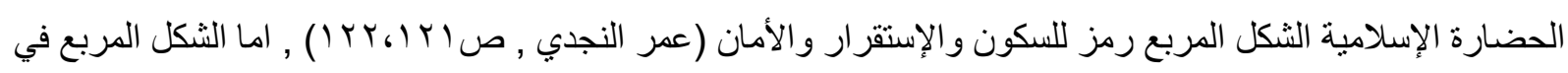
علم البايو جيومتري فهو شكل يحوي مختلف الذبذبات و الموجات ,فالمربع يتكون من خطين متعامدين ومتقاطعين في نقطة هذة النقطة هي مركز الإشعاع لأقصر واكثر الذبذبات قوة في العالم (تحليل الباحثة , من منطلق دورة تدريبية في علم "البايو جيومتري") - احتوى فراغ الدركاة على بابان يتوسطان الحائط الثمالي و الجنوبي من باب المدخل لينتقل مستخدم الفراغ إلى باقي

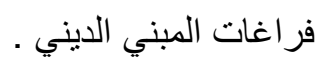
توصيات البحث - - موصي بدر اسة علاقة الدركاة بكتلة المدخل بمنشأة الحضارة الإسلامية بالعصر المملوكي البحري

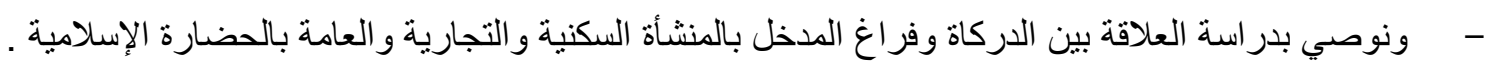
- كما نوصي بالإستفادة من منهج المصمم الإسلامي في تصميم المدخل الجانبي و الدركاة في التصميمات المعاصرة .

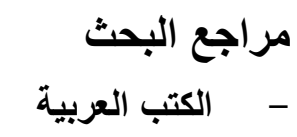
1- كريم أمير محمد ,أسرار الطاقة الحيوية والهندسة المقدة والتأمل ,دار أهل الحكمة للنشر,القاهرة , الطبعة الأولى $.5 r \cdot 1 r$, 1- karim 'amir muhamad, 'asrar altaaqat alhayawiat walhandasat almuqadasat walta'amul, dar 'ahl alhikmat lilnashr, alqahirat, altabeat al'uwlaa , $2012 \mathrm{mi}$.

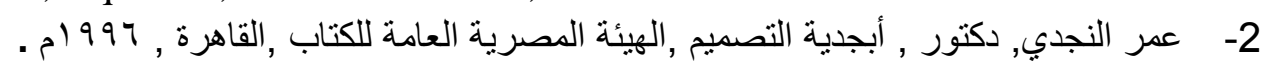

2- eumar alnajdi , duktur, 'abjadiat altasmim , alhayyat almisriat aleamat lilkitab , alqahirat , $1996 \mathrm{mi}$. 
3- مركز الدر اسات التخطيطية والمعمارية ,أسس التصميم المعماري و التخطيط الحضري في العصور الإسلامية المختلفة

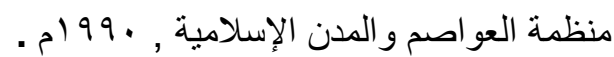

3- markaz aldirasat altakhtitiat walmiemariat, 'asas altasmim almiemaria waltakhtit aleumrania fi aleusur al'iislamiat almukhtalifat , munazamat aleawasim walmudun al'iislamiat , 1990 ma.

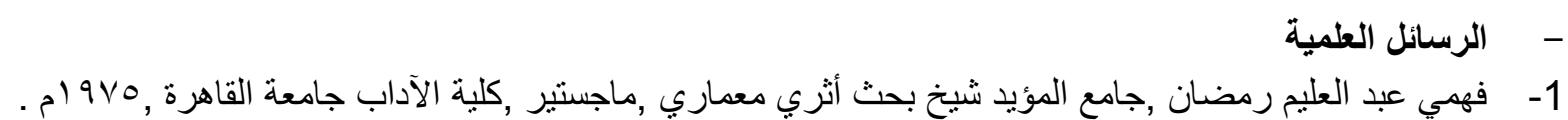
1- fahmi eabd alealim ramadan, jamie almuayid, bahth 'athariun wamiemariun, majistir , kuliyat aladab , jamieat alqahirat , $1975 \mathrm{mi}$.

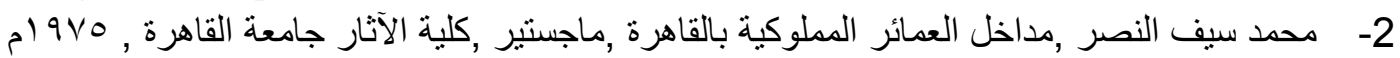

2- muhamad sayf alnasr, madakhil almabani almamlukiat bialqahirat, majistir, kuliyat aluathar , jamieat alqahirat, $1975 \mathrm{mi}$.

3- محمد فهيم , مدرسة السلطان قانصوة الغوري دراسة أثرية معمارية , ماجستير ,كلبة الآثار جامعة القاهرة ,

3- muhamad fahim , madrasat alsultan qunsuat alghuri , dirasat 'athariat wamiemariat, majistir , kuliyat aluathar , jamieat alqahirat , 1977.

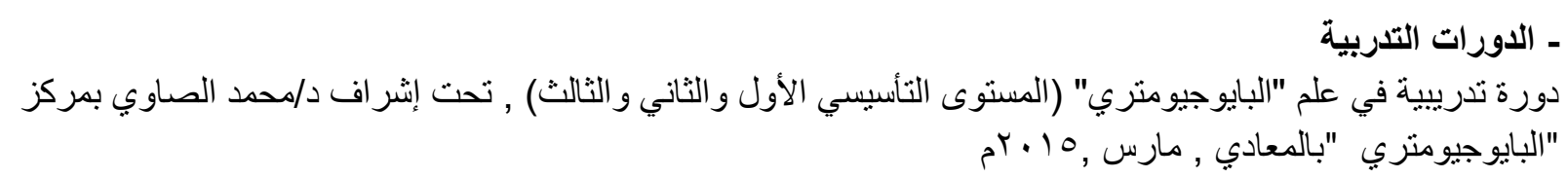
dawrat tadribiat fi eilam "albayujyumitri" (almustawaa altaasisii al'awal walthaani walthaalithi) taht 'iishraf d / muhamad alsaawi bimarkaz "albayujyumitri" bialmaeadi, mars, $2015 \mathrm{~m}$

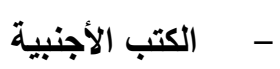

1- Ibrahim Karim ,Back to a future to mankind, BioGeometry Consulting,in Egypt 2009

2- Michael Erlewine, The Art Of Feng Shui ,2008 . 